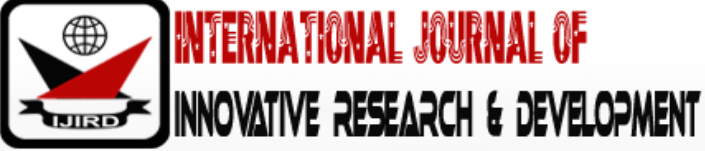

ISSN 2278 - 0211 (Online)

\section{Quantification of Fractures of Rock Analogue and Rock Analogue Mixed with Chemical Substance (Salt) Using Box-counting Method}

\author{
Wilson, M.C. \\ Lecturer, Department of Geological Engineering, \\ Kwame Nkrumah University of Science and Technology, Kumasi, Ghana
}

\begin{abstract}
:
The main aim of this paper is to quantify complex flow patterns of fractures of a mixture of raw starch and water and also that of starch, water and salt. The specific objectives are to: reveal the sort of fractures to be developed in the rock analogue, determine the effect of salt inclusion in the complex flow pattern, use the box-counting tool to quantify the complexity of the rock analogue patterns, and also deduce the significance of the quantification to geology.

Rock analogue is a structure that is similar in function to a rock but the chemical composition is different. The rock analogue used in this experiment was fine-grained starch. The experiment reveals the fractal nature in flow patterns as different forms of weight \% and different acts of viscosities and densities exhibit different forms and sizes of fractures. Different weights of starch were sampled with a specific and constant weight of $100 \mathrm{~g}$ of water. $10 \%$ weights of starch were sampled for the weight of salt. To quantify this flow experiment, two different methods of fractal geometry are applied, namely the MORFA and Box-counting. It could be observed that the higher and better the linear correlation, the lower the value of the fractal dimension and vice-versa. The average fractal dimension $\left(\mathrm{D}_{\mathrm{f}}\right.$ values for the fractures or cracks with salt (1.60) corresponds better to the $\mathrm{D}_{\mathrm{f}}$ values of Takayasu, NASA, Hirata, Barton and that of the general $\mathrm{D}_{\mathrm{f}}$ data for geophysics, than the $\mathrm{D}_{\mathrm{f}}$ values without salt (1.62).
\end{abstract}

Keywords: Rock analogue, viscosities, salt, fractal dimension, box-counting

\section{Introduction}

A structure can be represented partly or totally by a binary pattern. These patterns give revelation on the various properties of the material in question, for instance, the resistance to flow (viscosity), strength of the material, rate of chemical permeability in the material, etc. (Kruhl, 2012). Pattern is regarded as the geometric arrangement of two complimentary phases, for example, the distribution of black and white pixels in digitized images. Pattern quantification forms the basis for effective comparison of natural and experimental data and results of simulations (Peternell \& Kruhl, 2009).

Fractal geometry is one which is scale independent or self-similar at all scales between upper and lower fractal limits (Gillespie, et al., 1993). This scale-independent distribution is a power-law (fractal) distribution. The definition of a fractal distribution is that the number of objects $\mathrm{N}$ with a characteristic size greater than $\mathrm{r}$ scales with the relation $\mathrm{N}(\mathrm{r}) \sim \mathrm{r}$ -D. $\quad \rightarrow \mathrm{N}=\underline{\mathrm{C}} \mathrm{r}^{\mathrm{D}} \quad$......equation 1

The power determines the fractal dimension (of the coastline) and $\mathrm{C}$ a constant of proportionality (Mandelbrot, 1982).After the Mandelbrot's introduction of fractal geometry (Mandelbrot, 1977), a lot of geo-materials and their analogues, natural as well as artificial materials show fractal and these have been effectively quantified by methods of fractal geometry. In my experiments ofrock analogue (starch and also a mixture of starch and salt), two methods and software were applied. These include MORFA (Mapping of Rock Fabric Anisotropy) and BoxCounting.

This articleis divided into the experiment part to determine the different forms of viscosity and their flow pattern and the fractal nature and also the sizes and textures of the grains when they dry, analytical aspect with application of series of computer software programs to determine the anisotropy nature of a particular image and to distinguish the fractal dimension.

\section{Materials and Methods}

The whole experiment is to reveal the rate of flow of a raw complex rock analogue and a rock analogue diluted with some chemical substances such as salt and how to quantify them. Rock analogue is thus a structure that is similar in 
function to a rock but the origin or chemical composition is different. The rock analogue chosen in this wise was starch ("feine Speisestärke" in German market). Salt was used as chemical substance to mix with the starch in some of the experiments. I used a glass cup with labels of grams to measure the water, starch and salt contents in grams. A plastic substance was used to stir the starch, salt and water contents homogeneously (figure 1).

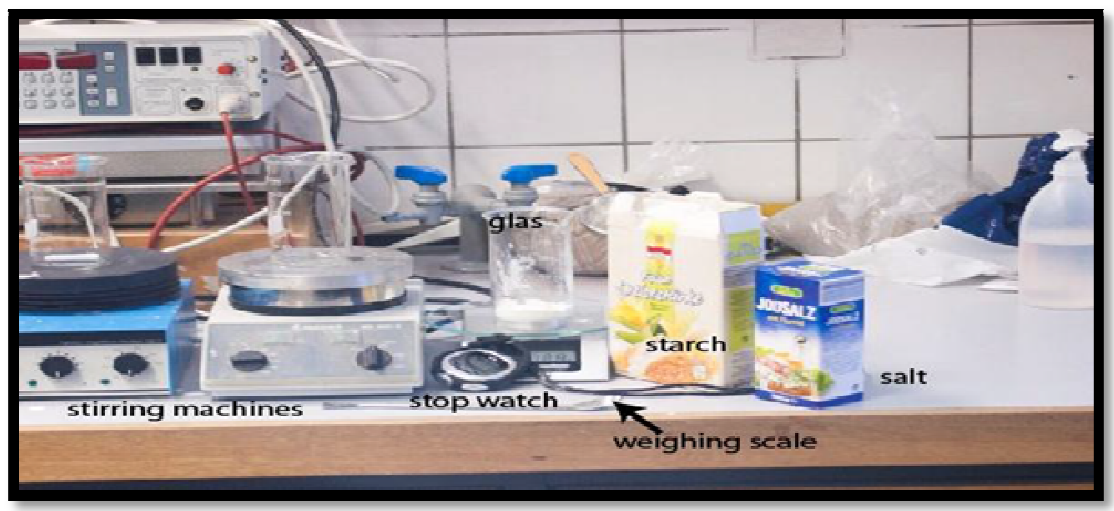

Figure 1: Apparatuses and Substances Used for the Experiment (Wilson, 2018)

A literal and constant weight of 100 grams of water was used throughout the experiments. However, different weight capacities of the rock analogue (starch) and salt were used to mix with the water. The weight of salt was set to be 10 weight \% of the starch content. Three different weight capacities of a mixture of raw starch and water and also a mixture of starch, salt and water were measured at three different viscosity rates (low, medium and high).

At low viscosity, $40 \mathrm{~g}$ of starch was measured, whilst $60 \mathrm{~g}$ and $80 \mathrm{~g}$ of starch were measured for medium and high viscosities respectively. Note that different forms of weight experiments were carried out before I could come out with this decision of $20 \mathrm{~g}$ intervals of different forms of viscosity. On considering the amount of salt concentration to mix with the starch, I considered the different weight percentages of the seas and that of oceans. For instance, the water of the dead seas has 33.7 weight \% of salt content (en.wikipedia.org)whilst that of the oceans account for only 3.5 weight $\%$ (sciencedaily.com). During my experiments I considered the weight of the water used to mix with the starch and the salt in each experiment (neither as much as the dead seas concentration nor low as that of the oceans), I literally chose 10 weight percent for my salt concentration. With respect to this, low viscosity mixture was set at $40 \mathrm{~g}$ and $4 \mathrm{~g}$ of starch and salt respectively, $60 \mathrm{~g}$ and $6 \mathrm{~g}$ of starch and salt for medium viscosity respectively, whilst the high viscosity recorded $80 \mathrm{~g}$ and $8 \mathrm{~g}$ of starch and salt respectively.

In performing the different forms of viscosity experiments, the low viscosity at $40 \mathrm{~g}$, the medium viscosity at $60 \mathrm{~g}$ and the high viscosity at $80 \mathrm{~g}$ of raw starch were weighed and added to the constant $100 \mathrm{~g}$ of water in a water cup containing a small plastic stirring tool. The contents were placed on the stirrer machine and allowed at different times to be homogeneously stirred. After the contents have been homogeneously stirred, the suspension (either raw starch or mixture of starch and salt) would be poured into the plastic bottle which was being held by two metallic clamps and the contents allowed at a free space to flow along the glass plate laid on the glass box (Figure 2). The distance between the glass plate and the plastic bottle (with the contents) was measured at approximately three millimeters.

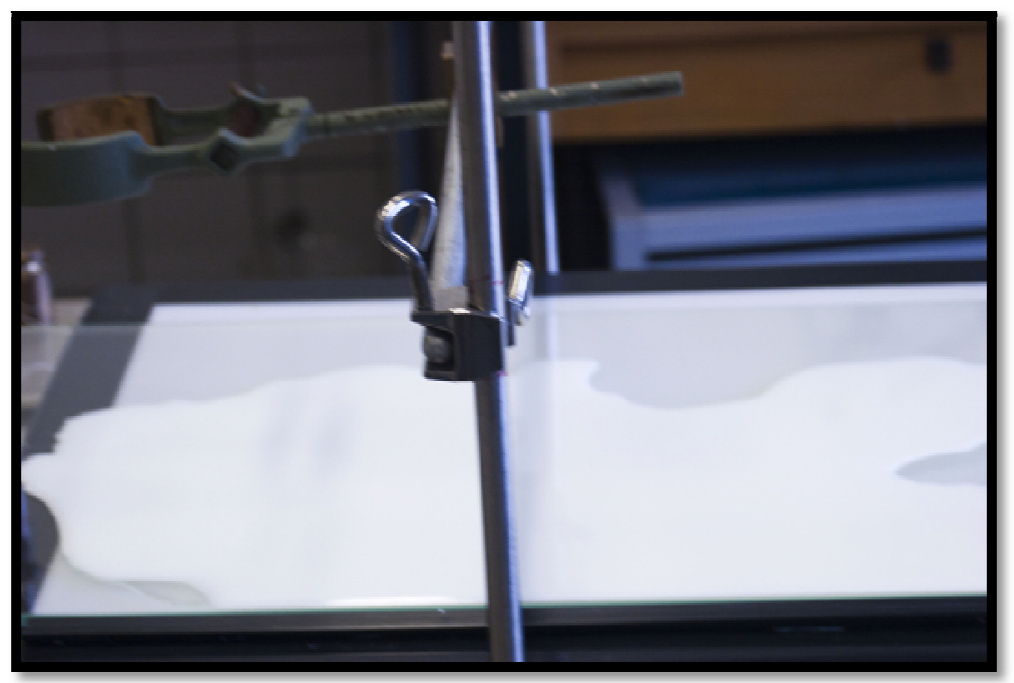

Figure 2: Flowing Starch and Salt Contents on a Glass Plate

\subsection{Stitching the Images}

The flow experiments took two days to dry up. The Nixon 70 camera was implored to take photographs of all the dried raw starch and the starch and salt mixtures. The camera was always set with the aid of two standing clamps on both 
sides of the light box (perpendicular to the clamp) over the viscous starch. An aperture of F11 and a time of 10 seconds were set for the camera to really search and take the needed and required photos. The chosen camera aperture of F11 and the set time of 10 seconds were set constant for all the photos taken for a better image resolution.

To be able to easily quantify the flow pattern, the photographing must be properly organized with a quality photographing resolution. For instance, if the camera aperture changes from one picture to the other or the timing or any other, there would be a bias of the pattern quantification. When the stitching is also not well done the pattern quantification would yield an equally biased or wrong result. Note thata very good quantification depends strictly on the quality of the pattern. These pictures would be set and analyzed from raw data (DSC NEF) to tiff images (DSC_tif) (Table 1) either with the PTGui Software or the Adobe Photoshop Software to get all the fifteen overlapped pictures stitched together as one packed clear picture. Each of the fifteen small pictures of a particular image represents only a single fraction of the total structure (Figure 3).

These parameters were set constant for all the experiments. This is because a slight change of any of these parameters and their resolutions would affect the quality of the pattern and such result in biasing of the results. For instance, color contrast between two different photos at different positions of the same experiment or different experiments lead to inaccuracy of the images. These fifteen photos were stitched together with the aidof Adobe Photoshop software. The photographs were stitched together and converted to grey-scale images in order to separate the starch and cracks of the rock analogue. To process the image begins with a clean- up using the Adobe Photoshop software (Peternell \& Kruhl, 2009).

\begin{tabular}{|c|c|c|c|c|c|}
\hline $\begin{array}{c}\text { Viscosity } \\
\text { Type }\end{array}$ & $\begin{array}{l}\text { Weight of } \\
\text { Water (g) }\end{array}$ & $\begin{array}{l}\text { Weight of } \\
\text { Starch (g) }\end{array}$ & $\begin{array}{c}\text { Weight of } \\
\text { Salt (g) }\end{array}$ & Raw Data & Tiff Image \\
\hline $\begin{array}{l}\text { Low/ } \\
\text { Medium/ } \\
\text { High }\end{array}$ & 100 & $40 / 60 / 80$ & $4 / 6 / 8$ & $\begin{array}{l}\text { DSC_3888.NEF } \\
\text { DSC_3889.NEF } \\
\text { DSC_3890.NEF } \\
\text { DSC_3891.NEF } \\
\text { DSC_3892.NEF } \\
\text { DSC_3893.NEF } \\
\text { DSC_3894.NEF } \\
\text { DSC_3895.NEF } \\
\text { DSC_3896.NEF } \\
\text { DSC_3897.NEF } \\
\text { DSC_3898.NEF } \\
\text { DSC_3899.NEF } \\
\text { DSC_3900.NEF } \\
\text { DSC_3901.NEF } \\
\text { DSC_3902.NEF }\end{array}$ & $\begin{array}{l}\text { DSC 3888.tif } \\
\text { DSC_3889.tif } \\
\text { DSC_3890.tif } \\
\text { DSC_3891.tif } \\
\text { DSC_3892.tif } \\
\text { DSC_3893.tif } \\
\text { DSC_3894.tif } \\
\text { DSC_3895.tif } \\
\text { DSC_3896.tif } \\
\text { DSC_3897.tif } \\
\text { DSC_3898.tif } \\
\text { DSC_3899.tif } \\
\text { DSC_3900.tif } \\
\text { DSC_3901.tif } \\
\text { DSC 3902.tif }\end{array}$ \\
\hline
\end{tabular}

Table 1: A Flow Experiment of a Mixture of Starch and Salt

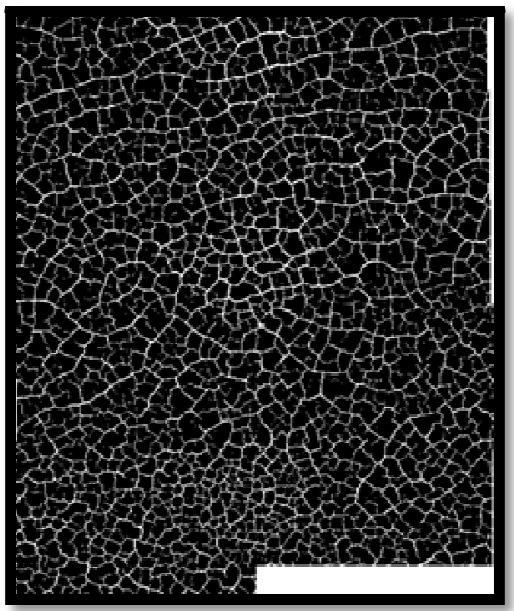

Figure 3: An Image Pattern of Fifteen Single Portions of Five Horizontal and Three Vertical Photos Stitched Together

The completely stitched images are then analyzed in the white and black phases to map the anisotropy of the pattern or fabric using MORFA (Mapping of Rock Forming Anisotropy) software. In this mapping procedure, gliding of the window is applied to the distribution of the pattern.

With MORFA analysis towards the quantification of anisotropic structures, various portions of height and width images were analyzed from the complete pattern (ranging from 1200 to 200 in my case of experiment). That means the box size of each image was defined from the largest image crop possible. The box size of the largest image crop was reduced until the results became unstable. Image for each viscosity was tested and the MORFA software made to run. 
The following points were applied to select good sub-image figures to be analyzed anisotropic with MORFA ANALYZE:

- The direction of the image should not be widely different from that of the others

- The correlation coefficient $\left(\mathrm{R}^{2}\right)$ of the ellipse or sphere should be high, at least $80 \%$

- The correlation coefficient $\left(\mathrm{R}^{2}\right)$ of the corresponding cumulative segment length points should as well be high, at least $80 \%$

- Good particle or point distribution on the ellipse(Wilson, 2018).

The larger the image height and width, the fewer the sub-images to be analyzed and vice-versa(Wilson, 2018).The Table 2 is the calibration table for the sub-images of figure 4 . The sub-images of the results were plotted using semi-log plot.

\begin{tabular}{|c|c|c|c|c|c|}
\hline Name & Description & $\begin{array}{c}\text { Heigth/Width } \\
\mathbf{( P x )}\end{array}$ & Orientation $\mathbf{(}^{\mathbf{O})}$ & $\begin{array}{c}\text { Threshold } \\
\text { Length }\end{array}$ & $\begin{array}{c}\text { Det. Coefficient } \\
\left.\mathbf{R}^{\mathbf{2}} \mathbf{\%}\right)\end{array}$ \\
\hline $60 \mathrm{~g}$ & Medium & $400 * 400$ & 134 & $20-80$ & 50 \\
raw & viscosity starch & $500 * 500$ & 143 & $20-80$ & 63 \\
starch & mixed with & $600 * 600$ & 31 & $20-80$ & 70 \\
& water & $700 * 700$ & 16 & $20-80$ & 89 \\
& & $800 * 800$ & 16 & $20-100$ & 89 \\
& & $900 * 900$ & 172 & $20-100$ & 95 \\
& & $1000 * 1000$ & 12 & $20-100$ & 92 \\
& & $1100 * 1100$ & 44 & $20-100$ & 49 \\
& & $1200 * 1200$ & 73 & $20-100$ & 88 \\
\hline
\end{tabular}

Table 2: Calibration Table of Sub-Images for MORFA Analyze

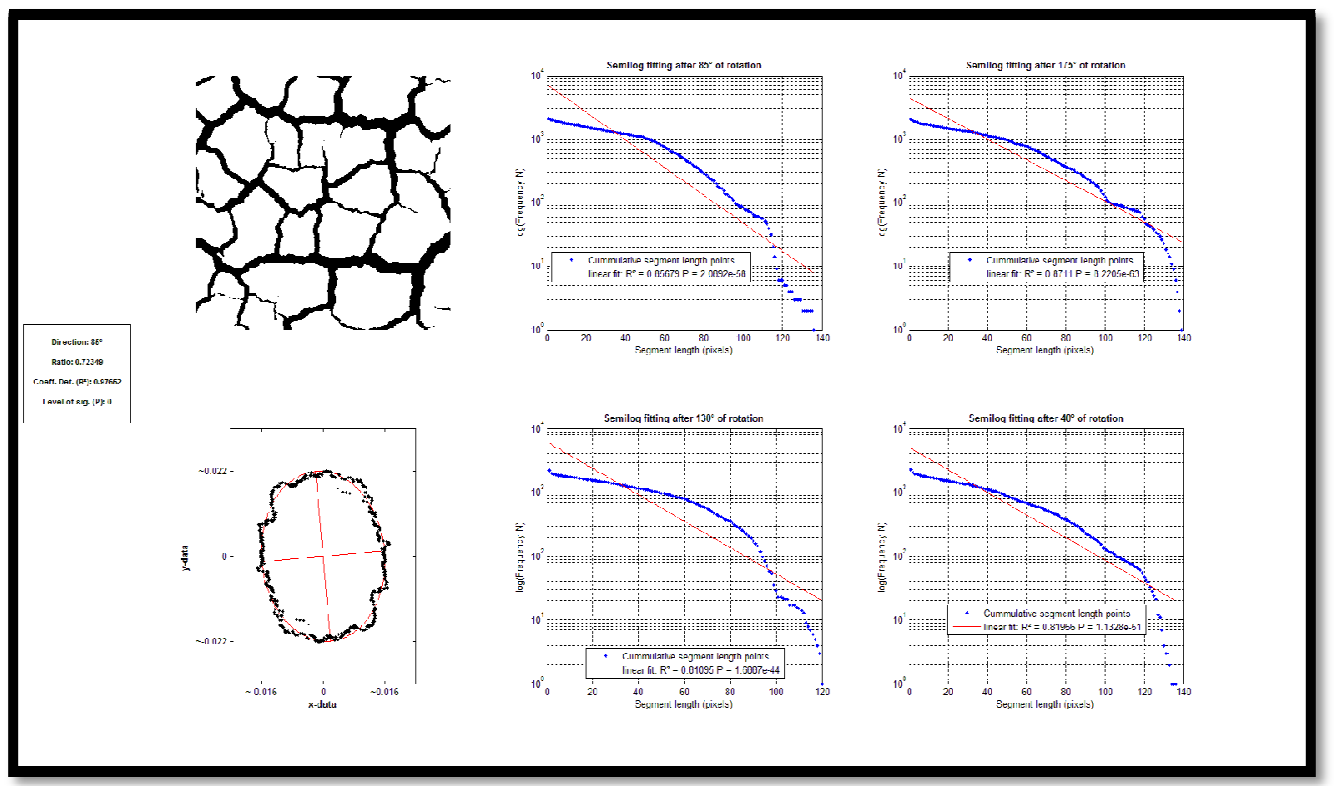

Figure 4: Semi-Log Sub-Image Plot of Low Viscosity Starch (White Phase =Cracks / Fractures)

The next step is to run the MORFA with the settings for all images and for both black (starch) and white (cracks / fractures) phases. Range of overlap for the various heights and widths of the boxes were set (Wilson, 2018). Fabrics in geo-materials and their related analogues (starch and salt) reveal patterns of complex nature. Their sizes, shapes as well as their directions also give information on the physical properties of materials. These fabrics which are mostly anisotropic in direction could be quantified. To analyze and quantify the anisotropy, note must be considered that irregular areas of analysis lead to different length of scan-lines in different directions which can falsify the overall result of quantifying the anisotropy.

The anisotropic directions exhibit the rate of deformation and its flow process. Anisotropy through deformation provides important information about structure forming processes and can be interpreted to be strain. Deformation processes relate to both a flow plane as well as a flow direction (figure 5). 


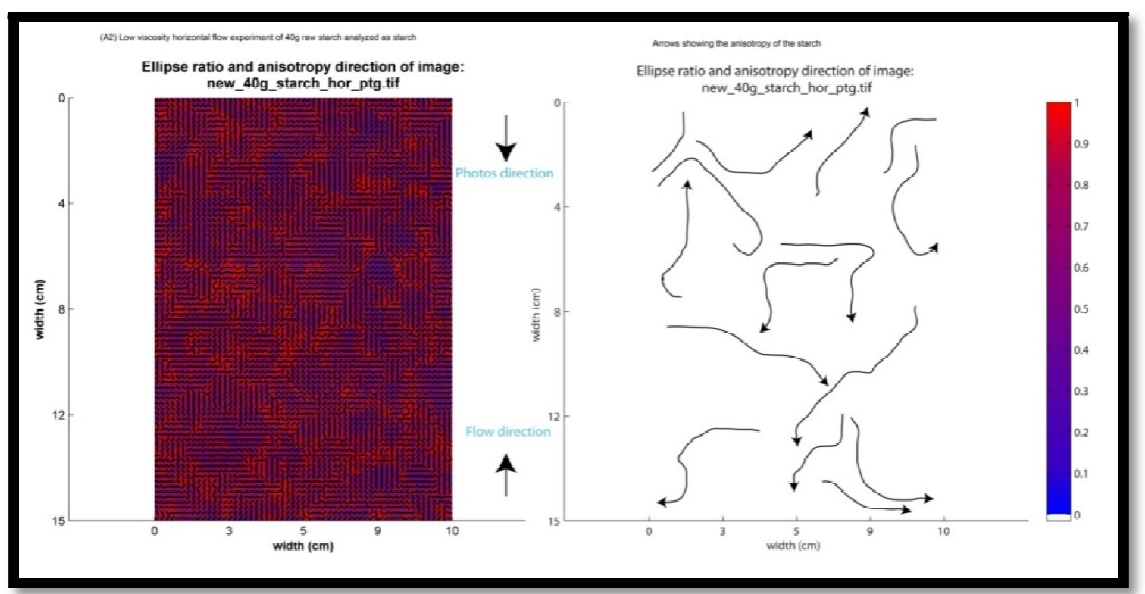

Figure 5: Low Viscosity Black Phase MORFA Image of A 40g Raw Starch at Horizontal Flow Analyzed as Starch with Its Anisotropy (Flow Direction)

To be able to really quantify anisotropy or pattern inhomogeneity, box counting is defined and applied together with window gliding method (Kruhl, 2012). This leads to quantification of fabrics or patterns of rock analogue. This method of fractal analysis was applied to the cracks and starch patterns to establish the fractal nature of the starch and crack pattern of the rock analogue. In determining the fractal geometry of this geological analogue, the box-counting method was applied.

Box-counting dimension $\left(D_{b}\right)$ which is a positive real number is defined by the relation $N(r) \sim r$ exp $D_{b}$; equation (2) where $D_{b}=$ box-counting dimension.

$\mathrm{N}(\mathrm{r})$ is the minimum number of boxes of length $\mathrm{r}$ necessary to cover a 2D pattern. For Euclidean objects, equation (2) defines $D_{b}=1$ for a straight line and 2 for a plane (Peternell \& Kruhl, 2009) $1<D_{b}<2$.

This method gathers data and uses the gathered data to analyze complex patterns. The analysis is done by breaking the set of data or image into smaller box-shaped sizes. In order to estimate the fractal dimension of the experiment, the Euclidean space containing the image pattern would have to be divided into a grid of boxes of size $\mathrm{r}$. The boxes $\mathrm{N}(\mathrm{r})$ which are not empty are counted (Takayasu, 1990). The procedure is repeated with the pattern image at smaller sizes (r) and each time the associated non-empty boxes are counted. Various portions of height and width images were analyzed from the complete pattern (ranging from 1200 to 200 Pixels at 100 pixels interval, in this experiment). N(r) is plotted against the reciprocal size $(1 / \mathrm{r})$ of the squares in a double-logarithmic diagram at a scale of $1 \mathrm{~cm}=522$ pixel (figure 6$)$. The required slope defines the fractal dimension.

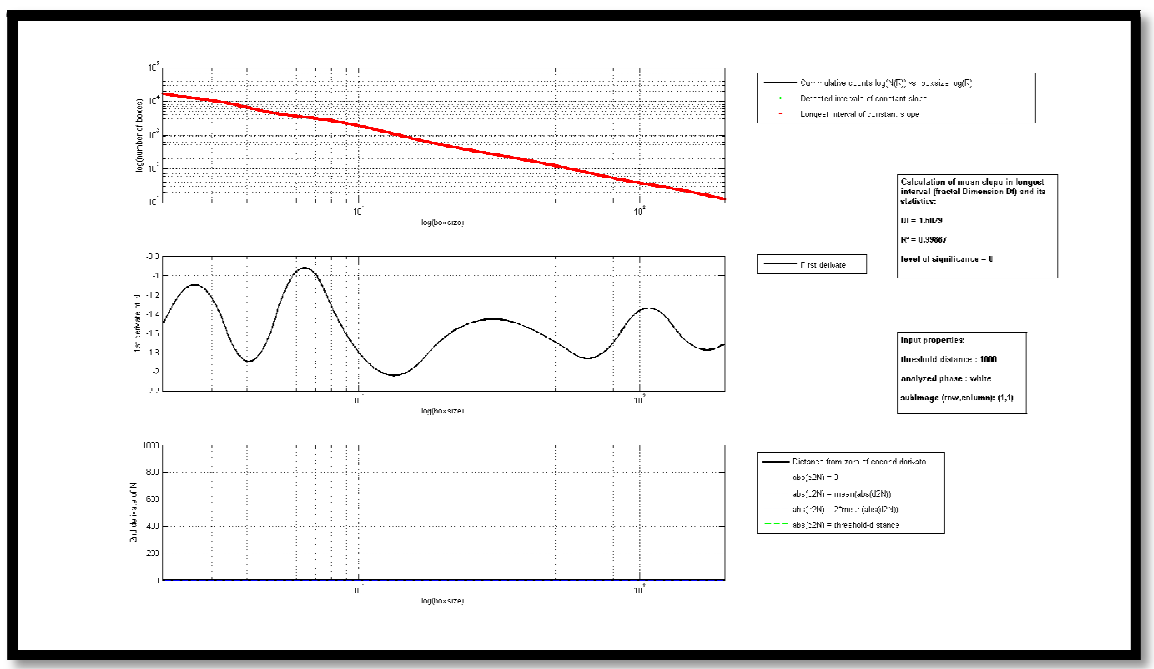

Figure 6: Box-Count High Viscosity Horizontal Experiment of 80g Starch and $8 \mathrm{~g}$ Salt (White Phase $=$ Cracks)

\section{Results and Discussion}

During the low viscosity experiment, the rate of flow was very fast. This means, less viscous leads to more flow. This low viscosity starch fluid could flow easily because its molecular makeup results in very little friction when it is in motion. The resistance to flow was very low. At the medium viscosity experiment, the flow rate was not fast. It needed time to flow. There was not much resistance to flow and only some water could flow out of it. For the high viscosity flow experiment (figures 7 and 8), the rate of flow was very slow. This was because the resistance to flow was very high [6]. It could be stated from here that: "thick fluids have higher viscosity than thin or runny fluids" (Fossen, 2010). The sizes of the cracks or fractures were differently observed with respect to the different forms of viscosity flow experiments. It was visibly observed that the low viscosity experiment at $40 \mathrm{~g}$ starch produced smaller cracks or fractures whilst the medium 
viscosity at $60 \mathrm{~g}$ starch and the high viscosity at $80 \mathrm{~g}$ starch experiments (figures 7 and 8) produced large and larger fractures respectively. The more viscous the fluid is, the bigger its size of molecules, structure and the cracks. All the lower viscosities have smaller sizes of molecules and thicknesses of cracks and structure. All the higher viscous fluids reveal larger molecular sizes as well as cracks, whilst the medium viscous fluids have their sizes lying between the lower and higher viscous fluids(Wilson, 2018).Mixing the starch and water together with a chemical substance, in this wise salt dilutes the mixture. Salt which has low porosity, low density and is impermeable, is itself viscous and behaves like a fluid (Fossen, 2010). Figure 8 reveals a darker complex image or background compared to the mixture without salt (figure 7). The sizes of the fractures or cracks of both the mixture with salt and that without salt (with respect to low, medium and high viscosities) are respectively small, big and bigger. However, the fracture sizes of the salt and starch mixture are bigger compared to the various fracture sizes of the different forms of viscosities without salt. Note that the weak and viscous nature of salt enables much wider areas to be deformed (Fossen, 2010). The different weights of salt give different deformational strengths. This is because the influence of salt during deformation depends on its thickness (Fossen, 2010).

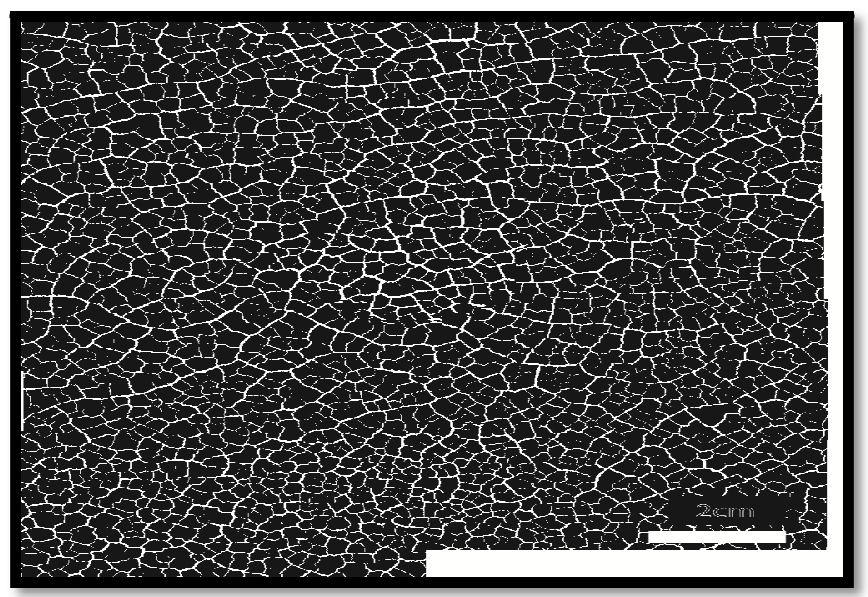

Figure 7: High Viscosity Horizontal Raw

Starch Experiment Of $80 \mathrm{~g}$



Figure 8: High Viscosity Horizontal Experiment of a

Mixture of $80 \mathrm{~g}$ of Starch and $8 \mathrm{~g}$ of Salt

In quantifying the anisotropy of the starch or the background (black phase) (figure 5) as well as the cracks or fractures (white phase) (figure 9) of both the mixture of starch and water and that of starch, water and salt with MORFA software, almost all the patterns exhibit anisotropic flow process. Anisotropy thus exhibits the rate of deformation and the strain of the ellipse and the deformation process. The flow direction is always from the inside to the outside and also in all the directions portraying an ellipsoidal shape. It could be observed from the various plotted images that the more the subimages leading to the anisotropy plots, the darker the face of the image. Considering the cracks (white phase analysis), the chosen height (Pixel) and width (pixel) with the corresponding threshold length and $\mathrm{R}^{2}$ portrayed fewer sub-images compared to most of the black phased images for starch. 


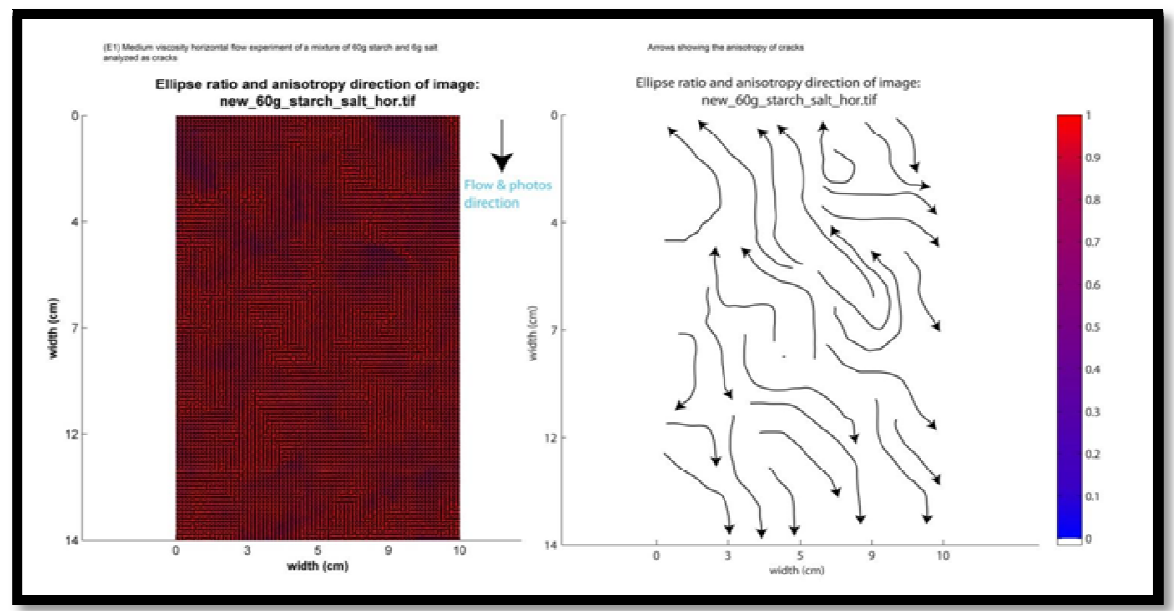

Figure 9: Medium viscosity horizontal flow experiment of MORFA results describing the anisotropy of the experiment of a mixture of $60 \mathrm{~g}$ starch and $6 \mathrm{~g}$ salt analysed asfractures

\subsection{Box-Counting Results and Discussions}

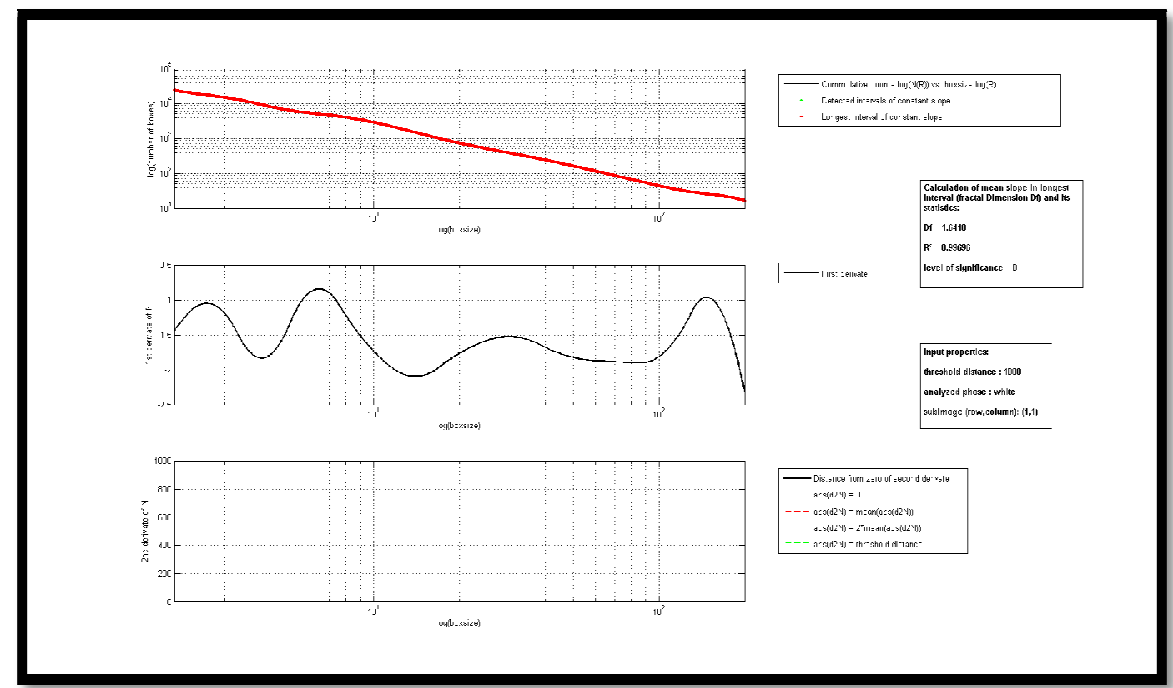

Figure 10: Box Count of Medium Viscosity Horizontal Flow Experiment at White Phase Analysis for Cracks or Fractures

With reference to figure 10 and Table 3 , the slope of the obtained straight line gives us the fractal dimension $\left(\mathrm{D}_{\mathrm{f}}\right)$ whilst the accuracy of these straight lines reveals the various correlation values $\left(R^{2}\right)$. Different ranges of values were detected for both the slope and the accuracy of the straight lines since different phases (white for cracks and black for starch) of analyses were conducted. Also, the addition of salt solutions to the starch revealed different values of slope $\left(D_{\mathrm{f}}\right)$ and accuracy $\left(\mathrm{R}^{2}\right)$.Straight lines with maximum correlation or fitting or accuracy with better slope were selected. It was generally observed that the correlation coefficients, $\mathrm{R}^{2}$ of the black phase in analyzing starch in the mixture of starch and water, ranged between 0.996 and 0.998 . The corresponding fractal dimension, $\mathrm{D}_{\mathrm{f}}$, of this black phase analysis ranged between 1.88 and 1.91. Also, the correlation coefficients, $\mathrm{R}^{2}$ of the black phase in analyzing starch in the mixture of starch, water and salt ranged between 0.997 and 0.998 . In other words, the black phase analysis gave a good linear correlation. However, the black phase analysis with the mixture of starch, water and salt gave a better linear correlation. The corresponding fractal dimension of this black phase analysis ranged between 1.84 and 1.89.With the white phase analysis, the correlation coefficient of the mixture of starch and water was seen to be constant at 0.996 with fractal dimension, $\mathrm{D}_{\mathrm{f}}$, ranging between 1.61 and 1.64. However, the correlation coefficient of starch, water and salt mixture flowing at a horizontal level revealed high values of 0.996 - 0.998, thus indicating a better linear correlation.

The $\mathrm{D}_{\mathrm{f}}$ for the fractures or cracks of the medium viscosity (1.64) was found to be higher than that of both the low and high viscosities of the mixture of water and starch. However, the $\mathrm{R}^{2}$ of the fractures of all the different classes of viscosities remained the same. Also, the $\mathrm{D}_{\mathrm{f}}$ of the starch (background) of the medium viscosity could be seen to be lower (1.88) than that of both the low and high viscosities of the same mixture of starch and water.

On the mixture of water, starch and salt, the $\mathrm{D}_{\mathrm{f}}$ of the fractures of the medium viscosity could be seen to be higher than that of the low and the high viscosities. However, the $\mathrm{D}_{\mathrm{f}}$ of the starch of the same mixture of medium viscosity could be seen to be lower than that of the low and high viscosities. Their $\mathrm{R}^{2}$ of all the three classes of viscosities remained the same. 


\begin{tabular}{|c|c|c|c|c|}
\hline Starch +water & $\begin{array}{c}\text { Low Viscosity } \\
\text { Medium Viscosity } \\
\text { High Viscosity }\end{array}$ & $\begin{array}{l}\text { Crack } \\
\text { Starch } \\
\text { Crack } \\
\text { Starch } \\
\text { crack } \\
\text { Starch }\end{array}$ & $\begin{array}{l}\mathrm{D}_{\mathrm{f}} \\
\mathrm{R}^{2} \\
\mathrm{D}_{\mathrm{f}} \\
\mathrm{R}^{2} \\
\mathrm{D}_{\mathrm{f}} \\
\mathrm{R}^{2} \\
\mathrm{D}_{\mathrm{f}} \\
\mathrm{R}^{2} \\
\mathrm{D}_{\mathrm{f}} \\
\mathrm{R}^{2} \\
\mathrm{D}_{\mathrm{f}} \\
\mathrm{R}^{2}\end{array}$ & $\begin{array}{c}1.61 \\
0.996 \\
1.91 \\
0.997 \\
1.64 \\
0.996 \\
1.88 \\
0.998 \\
1.61 \\
0.996 \\
1.90 \\
0.996\end{array}$ \\
\hline $\begin{array}{c}\text { Starch +Water + } \\
\text { Salt }\end{array}$ & $\begin{array}{c}\text { Low Viscosity } \\
\text { Medium Viscosity } \\
\text { High Viscosity }\end{array}$ & $\begin{array}{l}\text { Crack } \\
\text { Starch } \\
\text { Crack } \\
\text { Starch } \\
\text { Crack } \\
\text { Starch }\end{array}$ & $\begin{array}{l}D_{f} \\
R^{2} \\
D_{f} \\
R^{2} \\
D_{f} \\
R^{2} \\
D_{f} \\
R^{2} \\
D_{f} \\
R^{2} \\
D_{f} \\
R^{2}\end{array}$ & $\begin{array}{c}1.60 \\
0.996 \\
1.88 \\
0.998 \\
1.61 \\
0.997 \\
1.84 \\
0.998 \\
1.60 \\
0.998 \\
1.89 \\
0.998\end{array}$ \\
\hline
\end{tabular}

Table 3: Summarized Box-Counting Results

\subsection{Significance to Tectonic Activities}

The frequency-magnitude distribution of seismicity in Southern California from 1932 to 1994 has $\mathrm{D}_{\mathrm{f}}$ of 1.846 (Sieh et al., 1989). This helps in acquiring information on radiocarbon dating of faults and folds. (Barton, 1995)performed systematic studies on exposed joint and fault-trace patterns onYucca Mountain in Nevada. The fractal statistics were good correlated and the fractal dimensions ranged from 1.4 to 1.7. Measurements in geology and geophysics that yield power law spectra have their fractal behaviour lying between $1<\mathrm{D}<1.6$. (Takayasu, 1990) proposed the deterministic model of fracture, and ascertained the fractal dimension to be about 1.65 for the fracture pattern. The NASA Astrophysics Data System have indicated the fractal dimension $D_{\mathrm{f}}$ is about 1.5-1.6 at the central part of the Japan Arc, and decreases with distance from the centre. (Hirata, 1989) gave the fractal dimension of the fracture geometry of rocks as 1.6.

From my experiment, the box-count fractal dimension $D_{\mathrm{f}}$ values for starch or the background (in starch and water mixture) portrayed averagely 1.896 whilst the $\mathrm{D}_{\mathrm{f}}$ values for the fractures portrayed 1.62. The $\mathrm{D}_{\mathrm{f}}$ values for starch or the background (in the mixture of starch, water and salt) resulted in average 1.87 whilst the $\mathrm{D}_{\mathrm{f}}$ values for the fractures revealed 1.60.

\section{Conclusion}

Considering the low, medium and high viscous fluids in my experiment, the less viscous fluids lead to more flow and vice-versa. The molecular make-up plays an active role in the flow capacity. The lower the viscosity and density of the fluid, the lower the friction and resistance to flow resulting in easy flow and vice-versa. Also, the rate of viscosity and density of the fluid correspond to the size of fractures or cracks produced. Lower viscosity and lower density produce smaller cracks and vice-versa.

The inclusion of salt still provided higher values for the determination coefficients or correlation values $\left(\mathrm{R}^{2}\right)$ of crack analysis (white phase) at higher image heights and widths. However, the higher $\mathrm{R}^{2}$ values for starch analysis (black phase) were analyzed to fall at a moderate height and width of the image. Addition of salt to the water and starch mixture renders the background or image of the mixture dark. This implies that more salt reveals darker images of the complex flow, and thus the amount of salt in the starch and water mixture is directly proportional to the dark nature of the image. It could be seen that the quantity of starch in the mixture (with or without salt) defines the size of the fractures. Thus, $40 \mathrm{~g}$ starch at low viscosity portrays smaller fractures, whilst $60 \mathrm{~g}$ and $80 \mathrm{~g}$ starch at medium and high viscosities respectively portray big and bigger fractures. However, inclusion of salt in the mixture increases the sizes of fractures. This means, the rate of the salt content is directly proportional to the sizes of the fractures. This is due to the fact that the viscous nature of salt enables more wide areas to be deformed.

It could be generally observed that the anisotropy of both the cracks and the starch (though diverse directions) start from the inside and sprout out in multiple directions. Almost all the images are directed to both the top and down and sometimes to the other sides. Irrespective of how bright or dark the image is, all the images reveal multi anisotropic nature. However, the darker images, due to their many sub-images analysed, portray higher anisotropy.

It could be deduced from the box-counting results that the relatively high box-counting dimensions $\left(\mathrm{D}_{\mathrm{f}}\right)$ of 1.601.64 (white phase, fractures) and 1.84-1.91 (black phase, background) indicate a non-clustered distribution which argues for a homogenous crystallization history (Peternell \& Kruhl, 2009).

The $\mathrm{D}_{\mathrm{f}}$ of the mixture of starch and water of both the fracture and starch (background) are comparatively higher than that of the mixture of starch, water and salt. This is due to the fact that salt has low density, viscous and thus dilutes 
the mixture, hence, lower fractal dimension $\left(D_{\mathrm{f}}\right)$ values of both the starch and fractures. However, the correlation values or linear correlation $\left(R^{2}\right)$ of the mixture of water, salt and starch (0.996-0.998) is higher than that of only water and starch. This reveals that the higher and better the linear correlation $\left(\mathrm{R}^{2}\right)$, the lower the value of the fractal dimension $\left(\mathrm{D}_{\mathrm{f}}\right)$.

The average $D_{\mathrm{f}}$ value (1.87) for starch or background (in the mixture of water, starch and salt) could be seen to be closer to the $\mathrm{D}_{\mathrm{f}}$ of Sieh et al., 1989 (1.846) in the frequency-magnitude distribution of seismicity in Southern California from 1932 to 1994, than that of the mixture without salt. The $D_{\mathrm{f}}$ values for the fractures or cracks with salt (1.60) corresponds better to the $\mathrm{D}_{\mathrm{f}}$ values of Takayasu, NASA, Hirata, Barton and that of the general $\mathrm{D}_{\mathrm{f}}$ data for geophysics, than the $\mathrm{D}_{\mathrm{f}}$ values without salt (1.62). These values on earthquakes, joints and faults give revelation of the partings of weaknesses through which the magma flows to produce magmatic rocks. During intensive earthquake the crust undergoes deformation in the form of faulting or jointing. These places of weaknesses act as channel ways for the transmission of heat from the lower layers to the upper layers of the earth crust. This influx of heat from the lower layers causes considerable melting in the upper part of the crust. The rock material which is subsequently melted forms magmatic rocks. Magmatic rocks are formed from the cooling and subsequent solidification of hot molten rock material called magma.Magma migrates along these parting planes or planes of weakness such as fault or joint to the surface of the earth.

For Euclidean objects, $D_{\mathrm{f}}=1$ for a straight line and 2 for a plane (Peternell \& Kruhl, 2009).Both the starch and cracks of my experiments could be quantified to lie between 1 and 2. This means both the starch and cracks quantified values can be found between a line and a plane.The aforementioned measurements or values show a narrow range of fractal dimensions and thus do not indicate multi-fractal behavior and the structures are self-similar and also the process is a single pattern-forming process.

\section{Acknowledgement}

I am indeed grateful to Prof. Dr. Cees Passchier (head, Geologisches Institut, Johannes Gutenberg Universitaet Mainz, Germany) and Dr. Mark Peternell (senior lecturer, Universitaet Mainz, Germany) for wholeheartedly allowing me to use their geological laboratory to carry out my experiment.

\section{References}

i. Barton, C.C., 1995: Fractal analysis of scaling and spatial clustering of fractures. In: Barton, C.C., La Pointe, P.R. (Eds.), Fractals in the Earth Sciences. Plenum Press, New York,

ii. Fossen, H., 2010. Structural Geology. Cambridge University Press.

iii. Gillespie, P.A., Walsh, J.J., Howard, C.B., Waterson, J., 1993. Measurement and characterization of spatial distributions of fractures. Tectonophysics 226, 113-141

iv. Hirata, T., 1989. Fractal dimension of fault systems in Japan; Fractal structure in rock fracture geometry at various scales. Pure and Applied Geophysics 131 (1-2), 157-170.

v. https:/ / en.wikipedia.org/ wiki/Dead_Sea

vi. https:/ / www.sciencedaily.com/ terms/ seawater.htm

vii. Kruhl, J.H. 2012. Fractal geometry techniques in the quantification of complex rock structures: A special view on scaling regimes, inhomogeneity and anisotropy. Journal of Structural Geology xxx, 1-20

viii. Mandelbrot, B.B., 1977.Fractals - Form, Chance and Dimension. Freeman. San Francisco. Pp 365

ix. Mandelbrot, B.B. 1982. Fractal Geometry of Nature.

x. Peternell, M. \& Kruhl, J.H. 2009. Automation of pattern recognition and fractal geometry-based pattern quantification, exemplified by mineral-phase distribution patterns in igneous rocks. Computers and Geosciences 35, 1415-1426

xi. Sieh, K., Brillinger, D., Stuiver, M., 1989. A more precise chronology of earthquakes produced by the San Andreas Fault in southern California. Journal of geophysical research, 94.

xii. Takayasu, H., 1990. Fractals in the Physical Sciences. Manchester University Press, Manchester, pp. 170

xiii. Wilson, M.C. 2018. Quantification of fracture of rock analogue using a fractal geometry tool: Box-Counting. International Journal of Innovative Research and Development. Vol 7 Issue 4. DOI No.:10.24940 